\title{
Are anatomical landmark measurements accurate for predicting endotracheal tube depth?
}

\author{
Devanand Mangar, Collin J. Sprenker, Rachel A. Karlnoski, John P. McDonough, Robert K. Dodson, \\ Bill H. Brashears, Katheryne L. Downes and Enrico M. Camporesi*
}

\author{
Attending Anesthesiologist, Florida Gulf-to-Bay Anesthesiology Associates, 1 Tampa General Circle Suite A327, Tampa, USA \\ Research Coordinator, Florida Gulf-to-Bay Anesthesiology Associates, 1 Tampa General Circle Suite A327, Tampa, USA \\ Assistant Clinical Professor, Department of Surgery, University of South Florida, Tampa, FL University of North Florida, USA \\ Professor and Director, Nurse Anesthetist Program, 1 UNF Drive, Jacksonville, FL 32224, USA \\ Certified Registered Nurse Anesthetist, Florida Gulf-to-Bay Anesthesiology Associates, 1 Tampa General Circle Suite A327, Tampa, \\ USA \\ Certified Registered Nurse Anesthetist, Anesthesia Services of Benton County, P.A. 2710 Rife Medical Ln. Rogers, Arkansas, USA \\ Biostatistician, Biostatistics Core, University of South Florida, Tampa FL, USA \\ Emeritus Professor, Department of Surgery, University of South Florida, Tampa, FL University of North Florida, USA \\ *Corresponding author E-mail: ecampore@ health.usf.edu
}

\begin{abstract}
Background: A traditional method for depth of intubation in adult patients is taping the endotracheal tube (ETT) at the upper incisors a distance of 21 or $23 \mathrm{~cm}$ for women and men respectively (21/23 method). A novel "topographical" measurement for estimating proper intubation depth has been suggested as a better depth predictor compared to the $21 / 23$ method.
\end{abstract}

Objectives: To compare the 21/23 method to topographical measurements.

Methods: 100 ASA physical status I-II patients scheduled for elective surgery were enrolled. The 21/23 method was initially utilized for intubation and the ETT tip to carina distance was then fiberoptically measured. Anatomical (topographical) measurements were then obtained from each patient by adding the distance from the corner of the mouth to the mandibular angle to the distance from the mandibular angle to the center of the sternal notch and were recorded as the topographical intubation depth. Both measurements were assessed for percentage of ETTs that fell outside of our desired tip to carina range of $2-4 \mathrm{~cm}$.

Results: After the 21/23 method, $29 \%$ of ETTs fell outside our desired tip to carina range compared to $47 \%$ of ETTs with the topographical method $(\mathrm{p}=0.010)$. No correlation was found between the topographical measurements and airway length (upper incisors to vocal cords) ( $\mathrm{r}=0.248$ ).

Conclusions: Topographical measurements were not re-confirmed as an accurate intubation method and were found to be less reliable than the 21/23 method. We maintain that regardless of ETT insertion method utilized, the gold standard to ensure accurate ETT placement is fiberoptic inspection.

Keywords: Endotracheal tube, Intubation depth, anatomical measurements, fiberoptic inspection, malpositioned endotracheal tube

\section{Introduction}

The depth of tracheal intubation requires special precaution, as an ETT placed too deep can result in a collapsed lung with subsequent hypoxemia; conversely too shallow of an insertion may damage the vocal cords or become dislodged. It has been found that $39 \%$ of intubations result in complications and successful ETT placement on first attempt are highly correlated with level of experience [1].

Dronen et al. originally suggested that placing the ETT at a distance between 20 and $23 \mathrm{~cm}$, at the corner of the mouth, would result in fewer right mainstem bronchial intubations [2]. Following these guidelines, Owen et al established a simple method for the insertion of an ETT placing the tube at a depth of $21 \mathrm{~cm}$ and $23 \mathrm{~cm}$, at the upper incisor teeth, for 
women and men respectively [3]. This method however has revealed instances of endobronchial intubation and undesired tracheal depth [4-6].

A 2007 study by Evron et al. introduced the "topographical method", measuring anatomical landmarks, to estimate the proper ETT insertion depth [6]. The proposed benefit for this method is individual measurements accounting for anatomical variations of the head and neck versus a fixed measurement $(21$ or $23 \mathrm{~cm})$ based on gender. The $21 / 23$ method was compared to the topographical measurements for percentage of ETTs placed at a "best" tip position (4 cm above the carina). Superior results were found with the topographical method [6].

Polyurethane high-volume low-pressure (HVLP) cuffed endotracheal tubes (ETTs) are gaining wider use amongst clinicians. These ETTs feature longer cuffs made of ultrathin material $(10 \mu \mathrm{m})$ which reduce fluid and air leakage [7] and ventilator associated pneumonia [8-9], when compared to standard polyvinyl high-pressure low volume cuffed ETTs. Despite these benefits, our group has observed these ETTs significantly migrate from their fixed tracheal positions following neck flexion and extension [10]. These different cuff dimensions may affect the location of ETTs when utilizing previously applied intubation methods.

The goals of this study were to evaluate if topographical measurements are superior to the $21 / 23$ method in estimating proper ETT depth for tracheal intubation. The percentage of ETTs placed within our desired ETT tip to carina range of 2-4 cm was our main outcome. Secondarily, we prospectively evaluated the usage and efficacy of a HVLP polyurethane cuffed ETT with these two methods. We hypothesized that the previous findings with the topographical method could be reproduced, establishing a simple and effective intubation method.

\section{Materials and methods}

\subsection{Selection and description of participants}

The University of South Florida IRB approved the study protocol. All patients participating in the study signed a written informed consent. One hundred patients (50 males, 50 females) undergoing elective surgery, requiring routine intubation and general anesthesia at Tampa General Hospital, were enrolled in the study between February 2011- July 2011. Patients with upper airway or pulmonary complications, neck and/or chest distortion, previous difficult intubation, or patients at risk for pulmonary aspiration for gastric content were excluded from this study. All patients enrolled were American Society of Anesthesiologists (ASA) physical status I or II.

\subsection{Technical information}

Induction of anesthesia was obtained by administration of $2-3 \mathrm{mg} / \mathrm{kg}$ of propofol, $100 \mathrm{mg}$ of lidocaine, and $1.5 \mathrm{ug} / \mathrm{kg}$ of fentanyl. After induction, neuromuscular relaxation was obtained for intubation by administering $100 \mathrm{mg}$ of succinylcholine. Patients received mechanical ventilation with 1-2\% sevoflurane in oxygen-enriched air. Patients were placed supine, with the head and neck in the sniffing position, back and shoulders flush with the bed surface, and a folded towel under the occiput during intubation. Standard monitors were applied in the operating room to monitor vital signs, including blood pressure, heart rate, and oxygen saturation (SPO2).

All ETTs used were Kimberly Clark MicroCuff ${ }^{\circledR}$ ETT (Neenah, WI). All tubes used were uncut and had an internal diameter of $6.5,7,7.5$, or $8 \mathrm{~mm}$ (Table 1). ETT size was determined by the discretion of the anesthesiologist based on gender, patient height and weight.

\subsection{Study design}

After neuromuscular relaxation was obtained, all patients were intubated following the 21/23 method [11]: a depth of 21 or $23 \mathrm{~cm}$ for woman and men respectively measured at the upper incisors. After initial placement, the ETT tip to carina distance was measured fiberoptically. All ETTs were then repositioned to an "ideal" distance of $4 \mathrm{~cm}$ proximal to the carina, to standardize all patients to a safe tracheal depth, and left in place until extubation. Additionally, topographical measurements were obtained in all patients and recorded. These values were used to determine the insertion depth this method would place the ETT. The ETT tip to carina distance was calculated for each patient based off of the distance measured following the $21 / 23$ method. These distances were then compared to the 21/23 method for percentage of intubations that fell outside of our desired ETT tip to carina range of $2-4 \mathrm{~cm}$.

Additional data collected included: age, height, weight, body mass index (BMI), and Mallampati class (Table 1). The following fiberoptic measurements were also recorded: The distance of the vocal cords to the gum line at the incisors, the distance of the vocal cords to the carina and the top of ETT cuff to vocal cords after placement of the ETT at $4 \mathrm{~cm}$ 
above the carina (Table 2). The carina to vocal cords distance was visualized through the ETT by the fiberoptic bronchoscope.

\subsection{Method of topographical measurements}

With the ETT secured in place, measured anatomical landmarks were obtained, using a standard metric ruler $(\mathrm{cm})$, by the summation of: the distance from the mandibular angle to the corner of the mouth and the distance from the mandibular angle to the sternal notch (Fig.1).

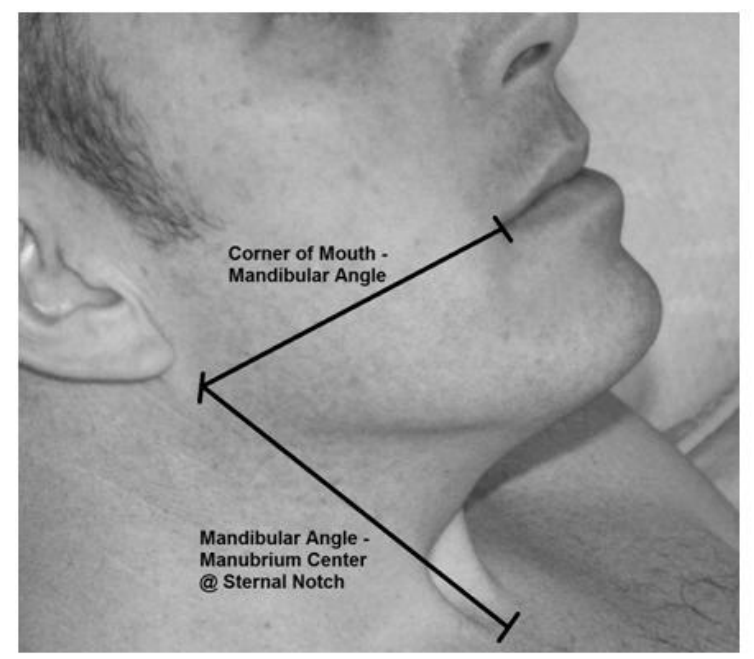

Fig 1: The topographical method is performed by summation of two measurements: 1) the distance from the corner of the mouth to the mandibular angle and 2) the distance from the mandibular angle to the manubrium center at the sternal notch.

\subsection{Measurement of ETT tip to carina distance}

To evaluate the ETT tip to carina distance, a fiberoptic bronchoscope (Olympus LF-GP, Center Valley, PA) and a standard metric ruler in centimeters $(\mathrm{cm})$ were used to determine the actual tip to carina distances (Fig. 2). All fiberoptic evaluations were performed by the same anesthesiologist (DM).

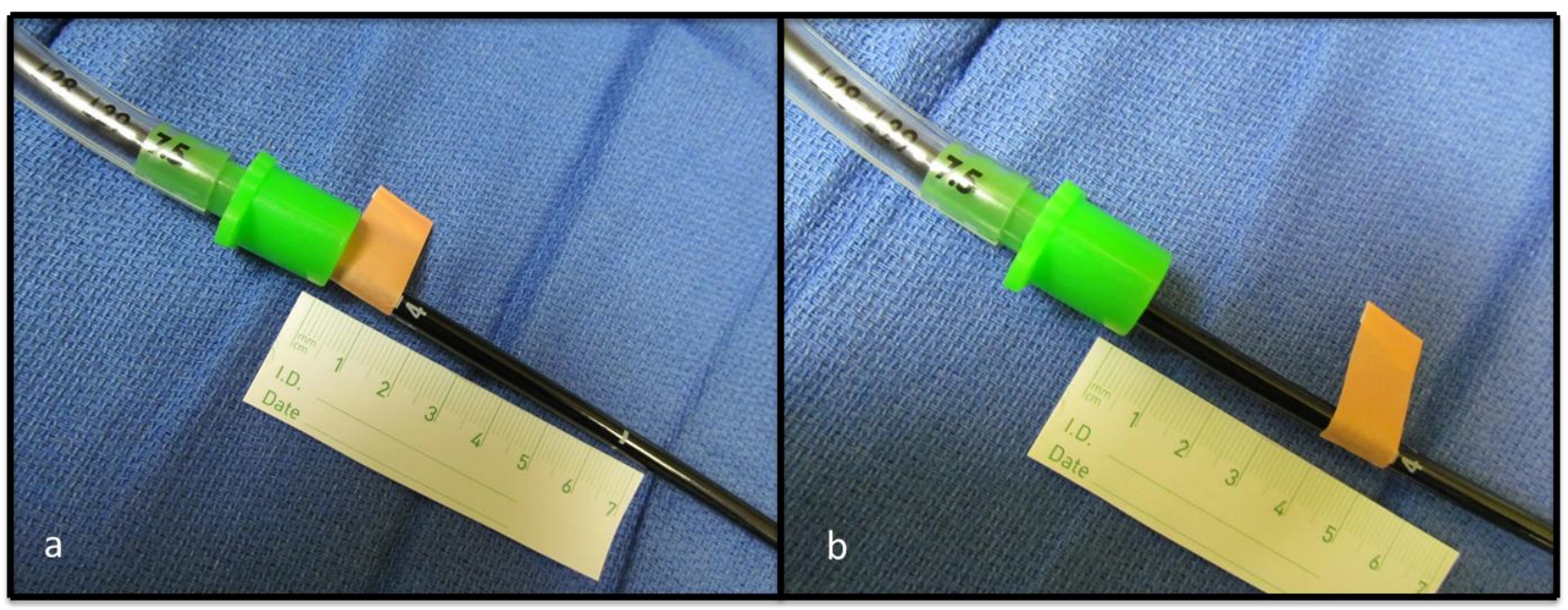

Fig 2: Method to measure the ETT tip to carina distance. The fiberoptic bronchoscope was inserted through the ETT with the tip touching the carina and a tape flag was placed to mark distance zero (Fig. 1a); The fiberoptic bronchoscope was moved to the position of the ETT tip and the displacement of the flag from the zero position measures the distance between the carina and the ETT tip on the centimeter scale (Fig. 1b). 
After final ETT placement, methods for confirmation of endotracheal intubation included auscultation for equal bilateral breath sounds, visual confirmation of bilaterally equal chest excursions, and end-tidal carbon dioxide assessment via capnography. Minimal occlusive volume of the ETT cuff was confirmed by an audible air leak at airway pressures greater than $20 \mathrm{cmH} 2 \mathrm{O}$.

\subsection{Statistical analysis}

Data were analyzed using SPSS 20.0 (IBM). All data between the 21/23 and topographical methods of endotracheal tube placement were analyzed to represent outcome comparisons. The normality and variance of the group distributions for continuous variables were first assessed using the overall Friedman's Test, followed by the Wilcoxon Signed Ranks test for related groups and the Mann-Whitney U or t-test for independent groups where appropriate. The McNemar paired proportion test was used to compare the percentage of intubations that fell outside of the desired range between the 21/23 method and topographical measurements. The topographical methods were compared to the 21/23 method using a single sample test of the values 21 and 23 (for women and men, respectively). Finally, the Chi-Square test was used to compare the gender groups. Continuous variables and intubation depth by method are expressed as mean \pm standard deviation and 95\% Confidence Intervals where appropriate. Intubation depths by methods are represented as mean \pm standard deviation (95\% confidence interval). P Values $<0.05$ were considered statistically significant.

\subsection{Sample size calculation}

In the study by Evron and colleagues [6], a reported 20\% difference between the 21/23 and the topographical intubation methods was used to calculate the power for our study. Sample size calculations indicated that a total sample size of $\mathrm{n}=100$ would be necessary to have $80 \%$ power to detect a difference in means of -0.54 (assuming $21 / 23$ method mean= 2.7, topographical method=3.24 and a standard deviation of differences of 1.9) with an alpha level $=0.05$.

\section{Results}

\subsection{Demographic variables}

We prospectively collected data on 100 patients and all patients completed the study.

There was 1 bronchial intubation using the 21/23 method but no occurrences of aspiration observed. Airway assessments on all participants did not exhibit any complications associated with the HVLP polyurethane cuffed ETTs. There was no statistically significant difference with age, height, weight, or body mass index (BMI) between genders (Table 1).

Table 1: Comparisons of Variables by Gender

\begin{tabular}{|c|c|c|c|}
\hline & Women $(n=50)$ & Men $(n=50)$ & Total $(n=100)$ \\
\hline Age & $55.0 \pm 16.2$ & $54.5 \pm 16.5$ & $54.7 \pm 16.2$ \\
\hline Height $(\mathrm{cm})$ & $162.9 \pm 6.5$ & $178.4 \pm 5.6$ & $170.6 \pm 9.9$ \\
\hline Weight $(\mathrm{kg})$ & $73.3 \pm 18.7$ & $88.2 \pm 18.9$ & $80.8 \pm 20.1$ \\
\hline $\mathrm{BMI}\left(\mathrm{kg} / \mathrm{M}^{2}\right)$ & $27.6 \pm 6.8$ & $27.6 \pm 5.2$ & $27.6 \pm 6.0$ \\
\hline \multicolumn{4}{|l|}{ Mallampati Class (n) } \\
\hline 1 & 16 & 12 & 28 \\
\hline 2 & 28 & 29 & 57 \\
\hline 3 & 6 & 9 & 15 \\
\hline \multicolumn{4}{|l|}{ ETT Size (n) } \\
\hline 6.5 & 1 & 0 & 1 \\
\hline 7 & 39 & 2 & 41 \\
\hline 7.5 & 10 & 40 & 50 \\
\hline 8 & 0 & 8 & 8 \\
\hline Volume of cuff inflation to achieve MOV (ml) & $10.6 \pm 1.4$ & $11.2 \pm 2.3$ & $10.9 \pm 1.9$ \\
\hline
\end{tabular}

Data are expressed as mean $\pm \mathrm{SD}$; ETT $=$ endotracheal tube; $\mathrm{MOV}=$ minimal occlusive volume 


\subsection{Topographical measurements and fiberoptic measured values}

Table 2 represents topographical measurements and fiberoptic measured values. Topographical measurements from the corner of mouth to mandibular angle and total topographical distance were significantly larger in men than women (P $<0.001$ and $\mathrm{P}=0.005$, respectively). Distance from vocal cords to gum line, measured at incisors were also found to be significantly larger in men when compared to women $(\mathrm{P}=<0.001)$.

Table 2: Topographical and Fiberoptically Measured Values

\begin{tabular}{|c|c|c|c|}
\hline & Women $(n=50)$ & Men $(n=50)$ & $\mathrm{p}$ value \\
\hline \multicolumn{4}{|l|}{ Topographical $(\mathrm{cm})$} \\
\hline Corner of mouth - Mandibular Angle (cm) & $7.7 \pm .9$ & $8.3 \pm .8$ & $<0.001 *$ \\
\hline $\begin{array}{l}\text { Mandibular Angle- Manubrium Center at } \\
\text { Sternal Notch }(\mathrm{cm}) \text { in neutral position }\end{array}$ & $12.6 \pm 1.7$ & $13.0 \pm 1.6$ & 0.4 \\
\hline Total Topographical Distance ${ }^{1}(\mathrm{~cm})$ & $20.3 \pm 1.8$ & $21.3 \pm 1.6$ & $0.01 *$ \\
\hline \multicolumn{4}{|l|}{ Fiberoptic $(\mathrm{cm})$} \\
\hline Vocal cords to gum line at incisors & $12.4 \pm 1.6$ & $14.9 \pm 1.6$ & $<0.001^{*}$ \\
\hline Vocal cords to carina & $11.5 \pm 2.0$ & $10.8 \pm 2.0$ & 0.09 \\
\hline $\begin{array}{l}\text { Top of ETT cuff to vocal cords after ideal } \\
\text { placement }^{2}\end{array}$ & $1.4 \pm 0.5$ & $1.4 \pm .490$ & 0.78 \\
\hline
\end{tabular}

Results are expressed as mean \pm SD, ETT $=$ Endotracheal Tube, $1=$ Summation of Distance from Corner of Mouth to mandibular angle and mandibular angle to sternal notch, $2=$ Ideal Tube position $=4 \mathrm{~cm}$ from carina

We found no relationship between the topographical measurements and the distance between the upper incisors and the vocal cords, revealing no correlation (Fig. 3).

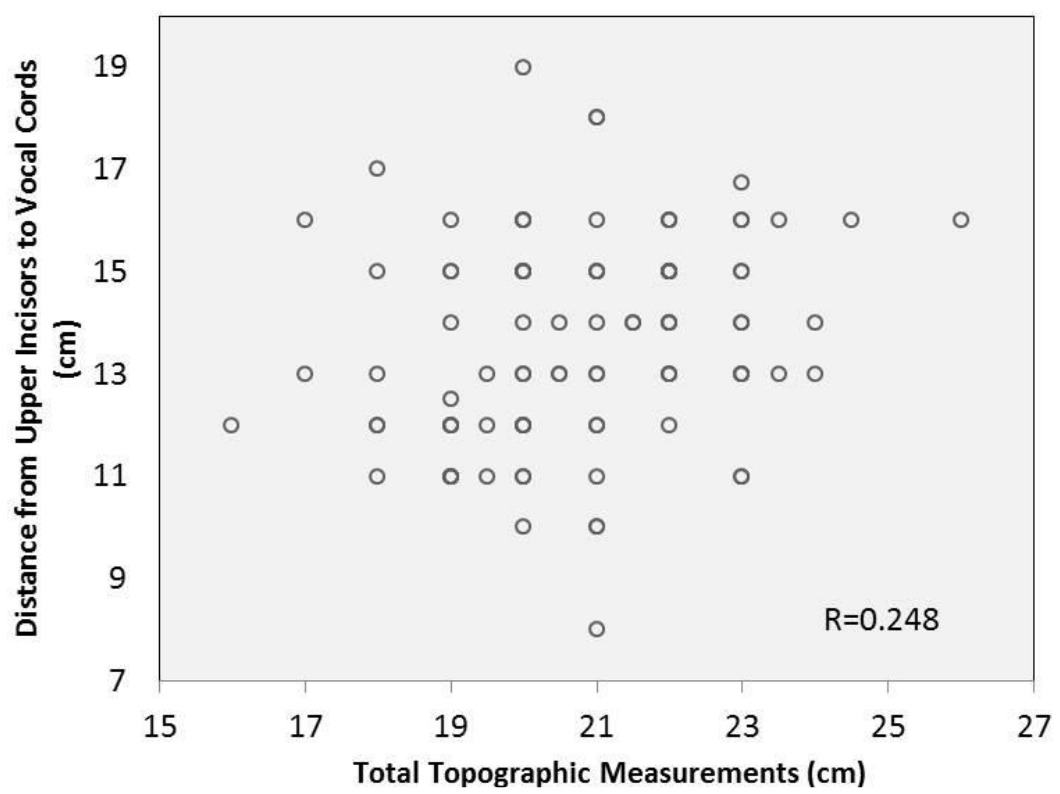

Fig 3: Scatter plot of the relationship between topographical measurements $(\mathrm{cm})$ and the distance between the upper incisors and the vocal cords $(\mathrm{cm})$. The Spearman's rank correlation coefficient $(\mathrm{R})$ was statistically significant $(\mathrm{P}<0.05)$.

\subsection{Intubation Depths}

Intubation depths and percentage of malpositioned ETTs are displayed in Table 3. The insertion depth was significantly different between the $21 / 23$ method and the topographical measurements for both women and men $(P=0.008$ and 
$<0.001$, respectively). Overall, the percentage of ETTs falling out of the desired tip to carina range was significantly different between the two methods ( $29 \%$ for $21 / 23$ vs. $47 \%$ for topographical, $\mathrm{p}=0.01$ ).

Table 3: Comparison of ETT depths by method

21/23 Topographical

Depth Measured at the upper incisors $(\mathrm{cm})$

\begin{tabular}{lccc}
\hline Female & 21 & $20.3 \pm 1.8(19.8-20.8)$ & $0.008^{*}$ \\
Male & 23 & $21.3 \pm 1.6(20.8-21.7)$ & $<0.001^{*}$ \\
Percent of intubations outside desired tip-carina range $(2-4 \mathrm{~cm})$ & & $0.010^{*}$ \\
\hline Overall & $29 \%$ & $47 \%$ & 0.143 \\
Female & $26 \%$ & $40 \%$ & 0.052 \\
Male & $32 \%$ & $54 \%$ & \\
\hline
\end{tabular}

Topographical Results expressed as mean $\pm \mathrm{SD}(95 \% \mathrm{CI}),{ }^{*}=\mathrm{p}<0.05$ is statistically significant

Figure 4 represents the distribution of the ETT tip to carina distance for the 21/23 method and topographical measurements.

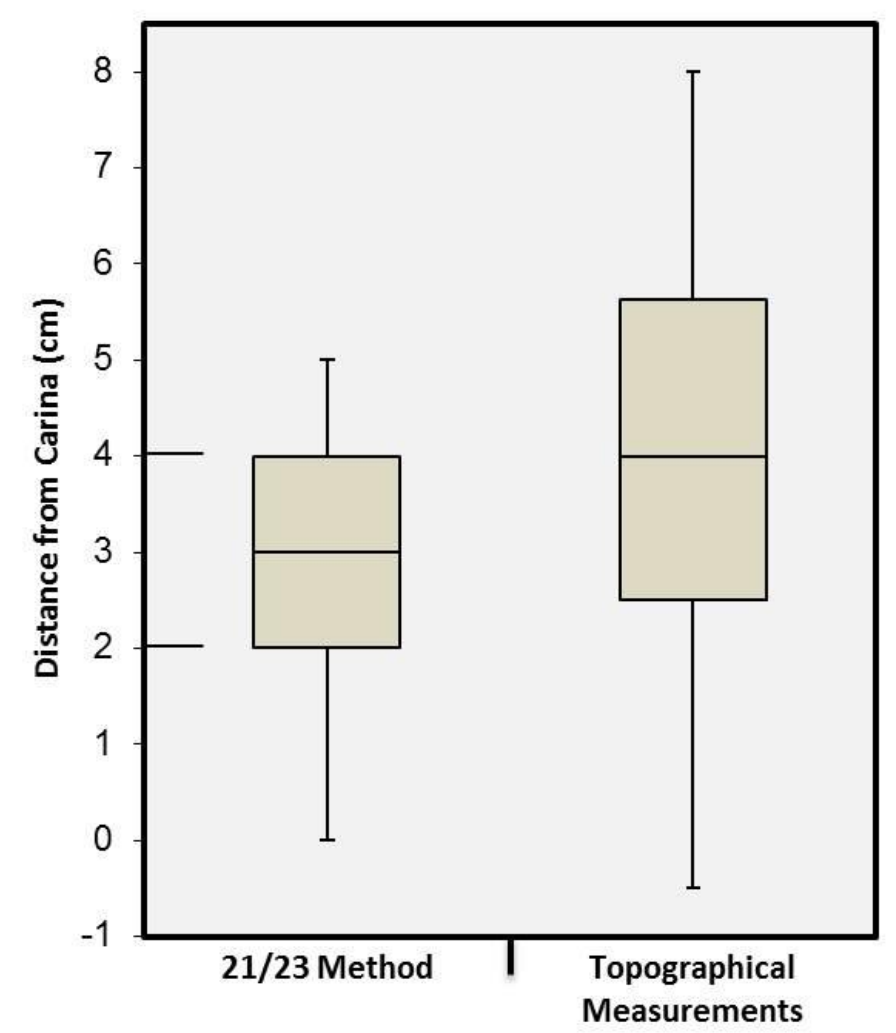

Fig 4: Box and whisker plot representing ETT tip to carina distances for all 100 patients for the 21/23 method and topographical measurements. The lines at 2 and $4 \mathrm{~cm}$ represent the desired ETT tip to carina distance used for the study. The ends of the whiskers are set at 1.5 times the Interquartile Range (IQR) above the third quartile (top of the box) and 1.5 times IQR below the first quartile (bottom of box). The line within the box represents the median value. 


\section{Discussion}

One major safety factor for mechanically ventilated patients is in the ability of ETTs to migrate in the trachea following patient movement [12-13]. Bronchial intubation following tube migration towards the carina or vocal cord impingement following the migration into the vocal cords may result from an improperly placed ETT.

Our study design was modeled on the study described by Evron et. al [6]. They performed a novel study that evaluated the topographical method as an alternative ETT insertion method. Following fiberoptic inspection, 58.5\% of ETT tips were placed too close to the carina $(<3 \mathrm{~cm})$ using the $21 / 23$ method; compared to $24 \%$ using topographic measurements $(P=0.0001)$ [6]. Despite these findings, the optimal ETT location was set to a "best fit" distance of $4 \mathrm{~cm}$ proximal to the carina. Our group elucidated this limiting factor by comparing these same two methods to a desired ETT tip to carina range of $2-4 \mathrm{~cm}$, which we believe to be more clinically relevant.

The current study failed to replicate the previously described benefits of external topographical landmarks as an accurate measurement for ETT placement. In fact, topographical measurements resulted in a significantly greater percentage of intubations outside the desired tip to carina range compared to the 21/23 method. We observed that the topographical measurements do not accurately replicate the ETTs pathway and may overestimate the desired intubation depth. Furthermore we found no correlation between topographical measurements and airway length (Fig. 3). We believe that increased neck size due to obesity limits the accuracy of this method.

We did not observe any complications with the HVLP ETTs used in this study. The 21/23 method placed the tubes in an acceptable range in most patients. The topographical measurements however had a large range in intubation depths (0.5-8 cm) (Figure 4). Had these depths been utilized, migration away from the carina would have likely induced vocal cord impingement, especially due to the longer cuff length of polyurethane HVLP ETTs [10].

In adults, multiple methods have been described for selecting the insertion level of an endotracheal tube (ETT) [3-5, 1421]. Owen et. al and Roberts et. al found positive outcomes with the 21/23 insertion method. Both studies analyzed critically ill patients and assessed the ETT position via chest x-ray. For the ideal ETT tip to carina distance, Owen et. al utilized $5 \pm 2 \mathrm{~cm}$ from the carina (Goodman's criteria) [22] to maintain a safe distance inside the trachea. The results revealed a significant reduction in endobronchial intubations $(\mathrm{p}<0.001)$ [3]. Conversely, Roberts et. al used $>2 \mathrm{~cm}$ from the carina for the proper placement of the ETT and concluded that placing the ETT according to the 21/23 method resulted in $97.6 \%$ correct placement [14]. More recent studies have found less beneficial results with this method suggesting this method should not be considered a more reliable method than the topographical measurements.

The occurrences of malpositioned ETTs have been correlated to patient height. In one study, the 21/23 method was evaluated in a shorter population [4]. Thirty-three percent of the intensive care patients had malpositioned ETTs in reference to Goodman's criteria. In 30 cases the ETT was noted to be $<3 \mathrm{~cm}$ from the carina. These 30 patients were found to be significantly shorter $(\mathrm{p}=0.01)$ when compared to the remaining study population. Cherng et. al [23] demonstrated a strong correlation with body height and airway length $(\mathrm{R}=0.7925$, carina to mouth angle height vs. body height) and derived a formula for obtaining optimal ETT length $[(\mathrm{cm})=$ (body height $(\mathrm{cm}) / 5)-13]$. Our study revealed only a moderate correlation between height and airway length $(R=0.510)$.

\section{Limitations}

A possible limitation to our measurements was not standardizing the ETT size. However, the majority of men ( $\mathrm{n}=40)$ utilized a size 7.5 ETT and the majority of women $(n=39)$ utilized a size 7 ETT (Table 1). In addition both insertion methods were evaluated on all tubes, thus expected impact is minimal. A second limitation is not actively intubating all patients based on the topographical measurements. However, we accurately calculated the correct tip to carina distance from the topographical measurements without unnecessarily re-intubating the patients.

\section{Conclusion}

We have demonstrated that neither the 21/23 method nor the topographical measurements are reliable for estimating proper ETT intubation depth. We therefore conclude that fixed distances are not accurate predictors for the proper placement of an ETT and add potential risk to patients. We maintain that the gold standard to ensure accurate ETT placement should be performed by placing the ETT cuff below the vocal cords following fiberoptic inspection. Perhaps it is most appropriate to quote the pioneers of the $21 / 23$ method when they said, "No method of doing anything in medicine is fool-proof." [24] 


\section{Conflict of interest statement}

The authors declare that there are no conflicts of interest.

\section{Acknowledgements}

No grants were obtained in relation to this paper

\section{References}

[1] Griesdale, Donald E. G., T. Laine Bosma, Tobias Kurth, George Isac, and Dean R. Chittock. "Complications of Endotracheal Intubation in the Critically Ill." Intensive Care Medicine 34.10 (2008): 1835-842

[2] Dronen S, Chadwick O, Nowak R: Endotracheal tip position in the arrested patient (letter). Ann Emerg Med 1982; 11:116-17.

[3] Owen RL, and FW Cheney. Endobronchial Intubation: A Preventable Complication. Anesthesiology 1987; 67:255-57.

[4] Ong, K. C, GD A'Court, P Eng, YY Ong: Ideal Endotracheal Tube Placement by Referencing Measurements on the Tube. Ann Acad Med Singapore 1996; $25.4: 550-52$.

[5] Hwang JY, Rhee KY, Kim JH, YS Park, SH Han: Methods of Endotracheal Tube Placement in Patients Undergoing Pelviscopic Surgery. Anaesth Intensive Care 2007; 35.6: 953-56.

[6] Evron S, Weisenberg M, Harow E, Khazin V, Szmuk P, Gavish D, T Ezri: "Proper Insertion Depth of Endotracheal Tubes in Adults by Topographic Landmarks Measurements." Journal of Clinical Anesthesia 19.1 (2007): 15-19.

[7] Dullenkopf A, Gerber A, Weiss M: Fluid leakage past tracheal tube cuffs: evaluation of the new Microcuff endotracheal tube. Intensive Care Med 2003; 29(10):1849-53.

[8] Poelaert J, Depuydt P, De Wolf A, Van de Velde S, Herck I, Blot S: Polyurethane cuffed endotracheal tubes to prevent early postoperative pneumonia after cardiac surgery: a pilot study. J Thorac Cardiovasc Surg 2008; 135(4):771-76.

[9] Miller MA, Arndt JL, Konkle MA, Chenoweth CE, Iwashyna TJ, Flaherty KR, Hyzy RC. A polyurethane cuffed endotracheal tube is associated with decreased rates of ventilator-associated pneumonia. J Crit Care. 2011 Jun;26(3):280-6. doi: 10.1016/j.jcrc.2010.05.035. Epub $2010 \mathrm{Jul} 10$

[10] Mangar D, Sprenker CJ, Karlnoski RA., Dodson R, Brashears B, Downes K, Camporesi EM. Migration of polyurethane high-volume lowpressure cuffed endotracheal tubes after neck flexion and extension. 2013 March. J of Anesth. [IN PRESS]

[11] Ovassapian A: Fiberoptic airway endoscopy in critical care, Fiberoptic endoscopy and the difficult airway, 2nd edition. Philadelphia, Lippincott-Raven Publishers, 1996, pp 164-6.

[12] Kim JT, Kim HJ, Ahn W, Kim HS, Bahk JH, Lee SC, Kim CS, SD Kim: Head Rotation, Flexion, and Extension Alter Endotracheal Tube Position in Adults and Children. Canadian Journal of Anesthesia 2009; 56.10 : 751-56.

[13] Hartrey R, IG Kestin: Movement of Oral and Nasal Tracheal Tubes as a Result of Changes in Head and Neck Position. Anaesthesia 1995; 50.8: 682-87.

[14] Roberts JR, Spadafora M, DC Cone: Proper Depth of Placement of Oral Endotracheal Tubes in Adults Prior to Radiographic Confirmation. Academic Emergency Medicine 1995; 2.1: 20-24.

[15] Techanivate A, Kumwilaisak K, S Samranrean: Estimation of the Proper Length of Orotracheal Intubation by Chula Formula. J Med Assoc Thai 2005; 88.12: 1838-846.

[16] Ong KC, GD A'Court, P Eng, YY Ong: Ideal Endotracheal Tube Placement by Referencing Measurements on the Tube. Ann Acad Med Singapore $1996 ; 25.4: 550-52$.

[17] Mehta, S: Intubation Guide Marks for Correct Tube Placement A Clinical Study. Anaesthesia 1991; 46.4: 306-08.

[18] Hwang JY, KY Rhee, JH Kim, YS Park, SH Han: Methods of Endotracheal Tube Placement in Patients Undergoing Pelviscopic Surgery. Anaesth Intensive Care 2007; 35.6: 953-56.

[19] Goldman JM, Armstrong J P, Vaught LE, LC Daniel: A New Method for Identifying the Depth of Insertion of Tracheal Tubes. Biomed Sci Instrum 1995; 31: 225-28.

[20] Ellis DG, Jakymec A, Kaplan RM, Stewart RD, Freeman JA, Bleyaert A, PE Berkebile: Guided Orotracheal Intubation in the Operating Room Using a Lighted Stylet: A Comparison with Direct Laryngoscopic Technique. Anesthesiology 1986; 64.6: 823-26.

[21] Patel N, Mahajan RP, and FR Ellis: Estimation of the Correct Length of Tracheal Tubes in Adults. Anaesthesia 1993; 48.1: 74-5.

[22] Goodman LR. Conrardy PA, Laing F, MM Singer: Radiographic Evaluation of Endotracheal Tube Position." Am J Roentgenol 1976; 127: 433-4.

[23] Cherng HC, Wong CS, Hsu CH, ST Ho: Airway length in adults: Estimation of the optimal endotracheal tube length for orotracheal intubation. Journal of Clinical Anesthesia, 2002; 14(4), 271-4.

[24] Cheney FW. A caution of the use of Routine Depth of insertion of Endotracheal Tubes (Letter). Anesthesiology 1991;74:961-962. 